\title{
Treatment of Lower Back Pain with Bone Marrow Concentrate
}

\author{
Marc Darrow* and Brent Shaw BS \\ Darrow Stem Cell Institute, USA
}

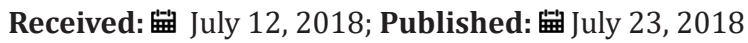

*Corresponding author: Marc Darrow, Darrow Stem Cell Institute, 11645 Wilshire Boulevard Suite 120, Los Angeles, CA 90025, USA

\begin{abstract}
Lower back pain can be a debilitating condition that is often caused by ligament and fascial sprains, and muscle strains. Even though the diagnosis may be a herniated disc, facet arthropathy, degenerative disc disease, spinal stenosis, scoliosis, spondylosis, spondylolisthesis, or other pathology, we have found for the past twenty years, with thousands of successfully treated patients, that the actual pain generator may not even be noted in the diagnosis. With the risk of adverse complications of surgery and ineffectiveness of epidural injections, bone marrow concentrate (BMC) offers a promising treatment to treat lower back pain. BMC contains mesenchymal stem cells that have the ability to differentiate into muscle, cartilage, and bone, in addition to releasing trophic factors that enhance tissue regeneration. The four patients included in this study underwent at least one treatment of BMC injection to the entheses of muscles, fascia, and ligaments surrounding the lumbar spine. At one-year follow-up all four patients experienced a decrease in resting and active pain. Patients also reported a mean $80 \%$ total overall improvement and were able to perform daily activities with less difficulty. These encouraging results warrant further investigation of the full potential of BMC injections for lower back pain.

Keywords: Stem Cells; Lower Back Pain; Non-Operative Treatment
\end{abstract}

\section{Introduction}

Lower back pain (LBP) is the second most debilitating condition in US [1] and is prevalent in $19.6 \%$ of adults between the ages of 20 and 59 [2]. Patients with chronic lower back pain experience limited range of motion, pain, and decreased quality of life. Muscle strains, ligament sprains, and muscle contusions account for up to 97\% of LBP in the adult population [3]. Additionally, researchers state that spinal ligaments are often neglected compared to other pathology that account for LBP [4]. This could be due to the overreliance of MRIs to guide physicians to correct diagnoses. Two studies illustrate that patients without symptomatic back pain displayed MRI abnormalities including degenerative disk changes $[5,6]$. Yet patients with these same diagnoses are recommended for surgery without a thorough understanding of their pain generator. Canadian researchers found that $55.7 \%$ of lumbar spine MRIs were considered inappropriate or of uncertain value to diagnosis [7].

Research is continually disproving prognosis tests in clinical application, including in recommendations for lumbar spinal fusion [8]. The drawbacks of patients undergoing unnecessary surgeries are the accompanied risks for adverse effect. Such procedures may cause serious complications such as dural tears, recurrent herniation, neurological problems, or even death [9]. A systematic review found that $16.4 \%$ of patients who underwent surgical back procedures experienced complications [10]. To avoid surgery, many patients receive steroid epidural injections for pain relief, however the efficacy of these injections have been shown to be limited and temporary if any [11]. The risk of side-effects and ineffectiveness of current treatments demonstrates a need for a noninvasive, conservative alternative to treat LBP.

Bone Marrow Concentrate (BMC) is a potential therapy to improve lower back patients' quality of life (Figure 1). BMC is a solution that contains many cytokines and growth factors but most notably contains platelets and mesenchymal stem cells (MSCs) [12]. MSCs are adult multipotent stem cells that have the ability to differentiate into different cell types such, cartilage, bone, and muscle [13]. This is a promising solution to tissue regeneration including regeneration of muscles and ligaments $[14,15]$. MSCs also secrete trophic factors which have anti-inflammatory properties and have been shown to promote muscle healing and growth through myogenesis of muscle progenitor cells [16-19]. Recently, MSCs were injected into patients with degenerative disc disease with reported improvement in disc quality, pain, and quality of life variables [20-22]. The patients in the present study were injected with BMC into the muscles, fascia, and ligaments surrounding the lumbar spine. It has been well documented in literature that MSCs and platelets enhance tendon and ligament healing [14,23]. In patients who were diagnosed with ligament and fascial sprains, we hypothesize that we can reduce patients' LBP by strengthening these anatomical areas. 
Lower back ligament sprains have been treated with dextrose prolotherapy for the past 80 years and patients have reported improvements in pain and function [24]. We hypothesize that since BMC has greater potential for tissue regeneration that patients could see even greater improvement. In addition, some LBP is generated from excess inflammation at the sites of tendon and ligament attachment. The MSCs and platelets in BMC have been shown to have natural anti-inflammatory properties [19,25] that would suppress this excess immune response and provide patients symptomatic relief. There are no current BMC studies that demonstrate any outcomes of this treatment approach. The objective of the present pilot study is to demonstrate that local lower back BMC injections can be an effective treatment protocol in treating LBP in an appropriate population.

\section{Methods}

\section{Patients}

This study reports a case series of clinical practice outcomes in which variables were administered prospectively and data was analyzed retrospectively. Patients who were included in this study underwent BMC treatments for lower back pain with at least a year follow-up. The procedures were done at a solo practitioner private practice from July 2016 to April 2017. All other patients who underwent BMC treatments for LBP without at least oneyear follow-up were excluded. All treatments were prescribed on an individual basis, as recommended by a physician. Written informed consent was obtained prior to each treatment. Three of the four patients required multiple BMC injections. If a patient at our clinic requires multiple injections, we direct them to receive injections approximately fourteen days apart, however scheduling conflicts often cause injection intervals more than fourteen days. At the fourteen day time period there is growth factor secretion from various cell types that participate in the late phases of wound healing $[26,27]$. Patients were instructed not to use non-steroidal anti-inflammatory drugs during treatment, since they hinder tissue regeneration [28]. Patient characteristics can be found in Tables $1 \& 2$. This study was constructed to follow all ethical guidelines directed by the Declaration of Helsinki.

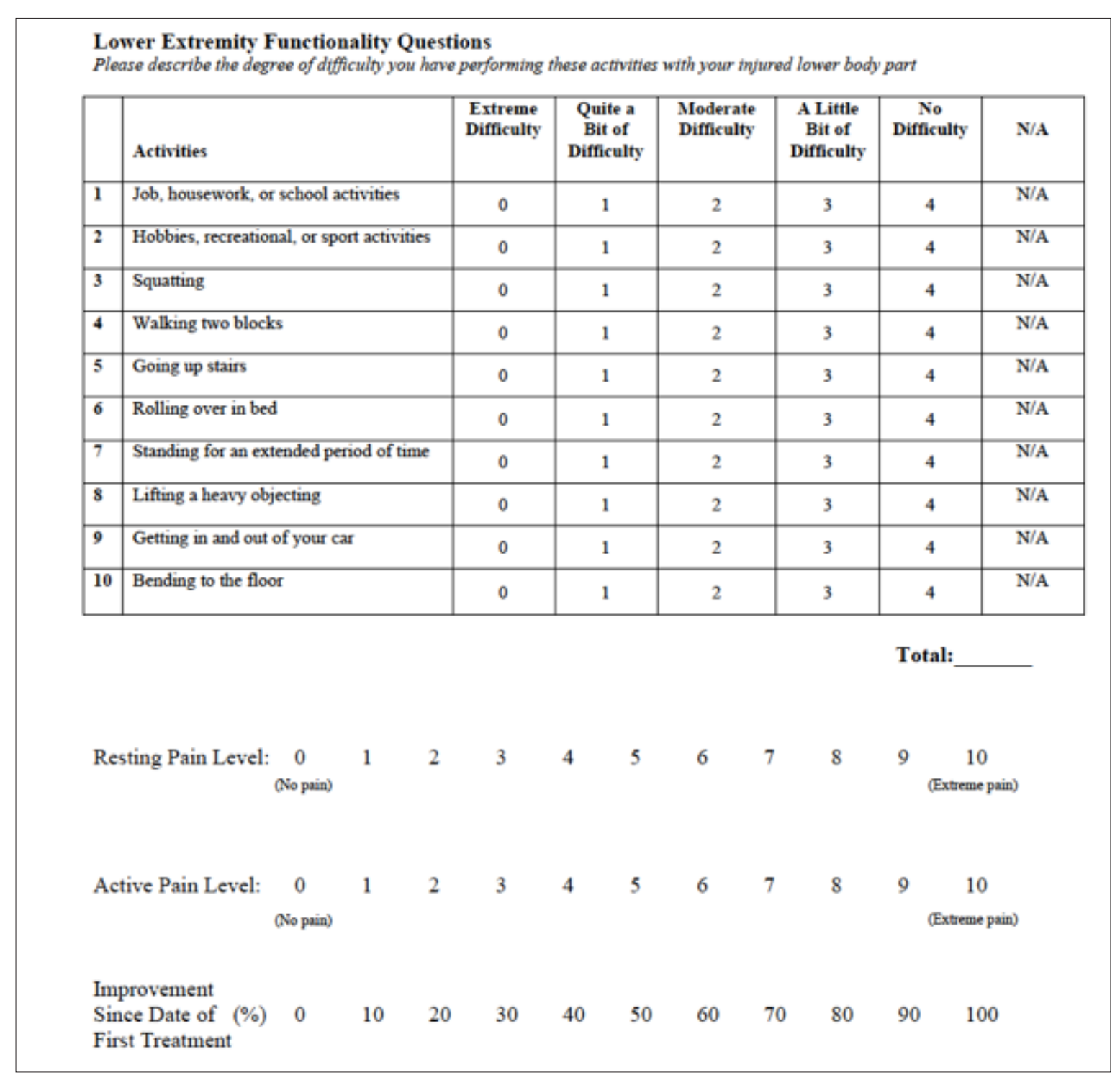

Figure 1 
Table 1: Patient Characteristics.

\begin{tabular}{|c|c|c|c|c|c|c|}
\hline Case Number & Sex & Age & BMI & Number of Treatments & Follow up after final injection & Pain Duration \\
\hline 1 & M & 65 & 23.01 & 2 & 1 year & 7 years \\
\hline 2 & M & 37 & 38.51 & 2 & 1 year & 2.5 years \\
\hline 3 & M & 56 & 27.37 & 4 & 1.5 year & 2 years \\
\hline 4 & F & 77 & 22.71 & 1 & 1 year & 20 years \\
\hline
\end{tabular}

\section{Procedure}

Patients were in the prone position and sterilized with $10 \%$ Povidone-Iodine on the skin above the posterior superior iliac spine (PSIS). Next, 4\% Chlorhexidine Gluconate (Hibiclens) was administered with sterile gauze in a circular motion starting at the PSIS. Patients were then anesthetized with $10 \mathrm{cc}$ of $1 \%$ lidocaine and 2 cc of $8.4 \%$ sodium bicarbonate, injected locally on and around the patient's posterior superior iliac spine. After sufficient local anesthesia was achieved, a fenestrated 11 gauge, 4-inch disposable needle was drilled to penetrate the PSIS and extract BMC. A 20-cc syringe prepared with $1 \mathrm{cc}$ of heparin (1,000 USP Units/cc) was used to extract BMC for a total yield of 19 cc. The needle was rotated slowly to capture the maximum number of MSCs within the ilium cavity and penetrated deeper as required, thereby minimizing peripheral blood in the sample. The BMC was then spun in a centrifuge, and the upper portion without visible red cells was isolated from the centrifuged BMC. 1-cc of Ropivacaine was added to 5-cc of centrifuged BMC to ensure less post-injection stiffness. Ropivacaine has shown limited toxicity to MSCs [29]. The injection sites were sterilized with $4 \%$ Hibiclens. The spun cells were injected by the physician into the tender or painful areas along the enthesis of the insertion of the quadratus lumborum, thoracodorsal fascia, iliac crest, interspinous and supraspinous ligaments, gluteus attachments to the pelvis, sacroiliac ligaments, etc., as determined per patient's complaints and tenderness.

\section{Outcomes}

The outcomes of interest in this study were changes to resting pain and active pain (numerical pain scale [NPS]), overall improvement (percentage scale), and function (scored questionnaire). Data was collected at baseline, at two-week followup after each treatment, and at least one year after the final injection. The functionality portion of the questionnaire, which assessed degree of difficulty in performing daily activities, was based on 10 of 20 activities assessed in the Lower Extremity Functional Scale [30] but also included a "not applicable (N/A)" response option. This scale has been shown to be a reliable functionality questionnaire for LBP [31]. The NPS to assess resting and active pain used a scale of 0 (no pain) to 10 (extreme pain) [32]. Lastly, the form included a subjective measure of how much overall improvement the patient experienced following treatment on a scale of $0 \%$ to $100 \%$.

\section{Cases}

\section{Case 1}

The first patient was a 65 year-old male with a seven-year history of lower back pain. His pain first occurred when he experienced intense cramping in his lower back while playing tennis. At one point because of difficulty walking, he went to the emergency room. The pain was most prevalent when running and sitting for extended periods of time. A radiograph of his lumbar spine demonstrated narrowing at L5-S1 and L3-L4 as well as grade I spondylolisthesis, of L5 on S1. However, after physical examination, the physician diagnosed the patient with a lumbosacral sprain.

The patient had undergone physical therapy, chiropractic adjustments, massage, and acupuncture that provided minimal pain relief. The lack of a conservative treatment had caused him to consider back surgery. His baseline characteristics were a resting pain of $2 / 10$, an active pain of $9 / 10$, and a functionality score of 20/40. The patient underwent 2 BMC treatments in 21 days. At short-term follow-up after the second treatment, the patient reported $60 \%$ total improvement, stating that he was in less pain and able to swim without difficulty. However, he continued to experience occasional stiffness. At the annual follow-up, patient reported a significant decrease in pain and was able to perform activities more easily. His final resting and active pain levels were $1 / 10$. He reported $80 \%$ total overall improvement, with a functionality score of $33 / 40$.

\section{Case 2}

The second patient was a 37-year-old male with a two and half year history of lower back pain. The patient had undergone chiropractic adjustments and massage therapy all of which provided only temporary relief. He underwent 4 Platelet-Rich Plasma (PRP) injections at another private practice, a year prior to BMC injection. The PRP injections provided no relief. It was then recommended that patient undergo surgery. Radiographic imaging of the patient's lumbar spine demonstrated disc bulges at L3-L4, L4-L5, and L5-S1, in addition to mild bilateral facet joint and ligamentum flavum hypertrophy. The pain was described as constant tightness that worsened when sitting for long periods or sleeping on his right side. After palpation of his lower back, it was clear that the pain was generated from a lumbosacral sprain. His baseline resting, and active pain were $4 / 10$ and functionality score was 28/40. The patient underwent 2 BMC treatments in 37 days. At the short-term follow-up after treatment, the patient reported a $60 \%$ overall improvement. He experienced increased flexibility, less pain, and was able to sleep better at night. A follow up was given a year after the conclusion of treatment and the patient continued to experience $60 \%$ improvement. His resting pain was $1 / 10$, active pain was $2 / 10$, and functionality was $32 / 40$.

\section{Case 3}

The third patient was a 56-year-old male who had a two-year history of lower back pain. The patient reported pain that was 
most prominent when sitting or lying on his stomach. MRI of his lumbar spine demonstrated moderate L3-L4 central stenosis due to a broad based disc bulge and facet degenerative change with hypertrophy. He underwent an epidural injection, physical therapy, and massage therapy none of which provided consistent pain relief. His resting pain was $3 / 10$, active pain was $6 / 10$, and functionality score was $17 / 40$ at baseline. When our physician examined his lower back, the patient was diagnosed with a lumbosacral sprain. The patient underwent four BMC treatments in a 146-day period. He experienced a minor improvement after the first treatment, reporting only dull aches with less frequency and stretching his lower back reduced the pain. After the third treatment, he reported improved ability to perform daily activities with less pain. At the short-term follow-up after the fourth treatment he experienced the most symptomatic relief, reporting a $75 \%$ total overall improvement. Another follow-up was administered approximately a year and half post-treatment, and his overall improvement increased to $90 \%$. Additionally, his resting pain was $0 / 10$, his active pain was $2 / 10$, and his functionality score increased to $34 / 40$.

Table 2: Patient Outcomes Scores.

\section{Case 4}

The fourth patient was a 77 year-old female with a 20 -year history of lower back pain, which had progressed with age. The patient wore a back brace to attempt to reduce the stiffness and pain when standing or sitting for extended periods of time. Radiographic assessment of her lumbar spine showed mild dextroscoliosis and mild narrowing of L1-L2, L3-L4 and moderately severe narrowing of L5-S1. Her baseline resting and active pain prior to treatment was $1 / 10$ and 5/10 respectively, and a 33/40 functionality score. After physical assessment of her lower back, our physician determined her pain was generated from a lumbosacral sprain. She had one BMC treatment and at first follow up two weeks after the injections, the patient experienced no pain or stiffness and reported $90 \%$ total improvement. Approximately a year after treatment, she felt even better, and stated that she was able to perform aerobics and line dancing for an hour and a half a day with no pain. She reported infrequent stiffness, but not as severe as it was prior to treatment. Her resting and active pain were $0 / 10$ and functionality score was $39 / 40$.

\begin{tabular}{|c|c|c|c|c|c|c|c|c|}
\hline Case Number & \multicolumn{2}{|c|}{ Resting Pain } & \multicolumn{2}{c|}{ Active Pain } & \multicolumn{2}{c|}{ Improvement Percentage } & \multicolumn{2}{c|}{ Functionality Score } \\
\hline & Pre & Post & Pre & Post & Pre & Post & Pre & Post \\
\hline 1 & 2 & 1 & 9 & 1 & $0 \%$ & $80 \%$ & 20 & 33 \\
\hline 2 & 4 & 1 & 4 & 2 & $0 \%$ & $60 \%$ & 28 & 32 \\
\hline 3 & 3 & 0 & 6 & 2 & $0 \%$ & $90 \%$ & 17 & 34 \\
\hline 4 & 1 & 0 & 5 & 0 & $0 \%$ & $90 \%$ & 33 & 39 \\
\hline Average & 2.5 & 0.5 & 6 & 1.3 & $0 \%$ & $80 \%$ & 24.5 & 34.5 \\
\hline
\end{tabular}

\section{Discussion}

This is the first reported study treating LBP with BMC injections to the ligaments, fascia, and muscles surrounding the lumbar spine. It is promising that at one-year follow-up, $100 \%$ of patients in this study experienced a decrease in resting and active pain in addition to performing daily activities with less difficulty. All four patients experienced sustained or increased improvement at annual follow-up compared to short-term follow-up. On average, patients reported an $80 \%$ decrease in resting pain, a $78 \%$ decrease in active pain, and a $41 \%$ increase in functionality score. Additionally, patients reported a mean $80 \%$ total overall improvement following treatment. The two patients who considered surgery prior to BMC treatment no longer felt the need for it. These results provide evidence that appropriately chosen patients with LBP may find relief with BMC injections.

The second patient provides a case where previous PRP injections at a separate treatment center failed to provide pain relief, but later BMC did provide relief. BMC which contains PRP, MSCs, and other growth factors is thought to be more therapeutic than PRP alone, however there are no comparative studies for treating lower back pain using these two treatments. Yet at follow up a year post BMC treatment, the patient continued to experience symptomatic relief. It has been reported in knee and hip OA patients that PRP is less effective in more severe pathology compared to mild cases $[33,34]$. We hypothesize this trend is also present in
LBP, which would explain why this patient experienced no benefit from PRP alone. Additional studies are needed to investigate the full potential and limitations of BMC and PRP treatments for LBP.

All published BMC studies for the lower back have highlighted intradiscal procedures for degenerative disc disease. One of these studies reported on five patients who were injected intradiscally with expanded numbers of MSCs [20]. These patients continued to experience improvement at the four to six years follow up [20]. Additionally, none of these patients experienced any adverse effects from the procedure.

It is promising that our patients who received non-intradiscal injections, and patients who received intradiscal injections, both continued to experience improvements with no adverse effects. The safety and efficacy of these treatments warrant further supplementary studies with better methods and larger samples sizes to validate these results. Studies that include intradiscal and non-intradiscal BMC injections are also needed to determine if combining these two treatments would provide more symptomatic relief than either treatment alone.

BMC is an attractive modality to treat LBP due to its low rate of adverse effects compared to surgery. A recent analysis of the adverse effects of same day BMC treatment for the knee, hip, ankle/ foot, hand/wrist, elbow, shoulder, and spine found only $0.4 \%$ of 1589 patients experienced a serious adverse event [35]. A serious 
adverse event was defined as "any untoward event that results in death, is life-threatening, requires inpatient hospitalization or causes prolongation of existing hospitalization, results in persistent or significant disability/incapacity, or requires intervention to prevent permanent impairment or damage [35,36]. This study stated that its reported rate of serious adverse events in this study was significantly lower when compared to those of surgery [35,37]. However injecting BMC intradiscally may bring upon unnecessary risks. Rare side effects such as neurological damage or spinal fluid leakage have been reported in patients who underwent injections via the epidural route [38]. These complications are avoided when injecting non-intradiscally, allowing patients less risk. The necessity of intradiscal injections may become obsolete by strengthening damaged or weakened ligaments through BMC injection. This would increase the stability of the spine, which would lower the frequency of discogenic abnormalities [39]. The shortcomings of this study are the small sample size, lack of control-randomization, and absence of a nucleated cell count.

The subjective nature of the variables measured may be prone to response bias. Further randomized-controlled studies with larger sample sizes are wanted to further the validity of these results.

\section{Conclusion}

All patients in this study experienced decreased pain and were able to perform daily activities with less difficulty after one-year follow-up. These encouraging results warrant further studies to explore the injection of BMC to the muscles, ligaments, and fascia surrounding the lumbar spine to relieve lower back pain

\section{Disclosure}

Marc Darrow is the only physician at Darrow Stem Cell Institute, where all study procedures were performed.

\section{References}

1. (2001) From the Centers for Disease Control and Prevention. Prevalence of disabilities and associated health conditions among adults--United States, 1999 JAMA 285(12): 1571-1572

2. Meucci RD, Fassa AG, Faria NMX (2015) Prevalence of chronic low back pain: systematic review. Revista de Saúde Pública 49: 1.

3. An HS, Jenis LG, Vaccaro AR (1999) Adult spine trauma. In Beaty JH (Eds.). Orthopaedic Knowledge Update 6. Rosemont, IL: American Academy of Orthopedic Surgeons pp. 653-671

4. Hammer N, Steinke H, Lingslebe U, Bechmann I, Josten C, et al. (2013) Ligamentous influence in pelvic load distribution. Spine J 13(10): 13211330.

5. Kovacs FM, Arana E (2016) Degenerative disease of the lumbar spine. Radiologia 58(1): 26-34.

6. Franz EW, Bentley JN, Yee PPS, et al. (2015) Patient misconceptions concerning lumbar spondylosis diagnosis and treatment. J Neurosurg Spine 22(5): 496-502.

7. Emery DJ, Shojania KG, Forster AJ, Mojaverian N, Feasby TE (2013) Overuse of Magnetic Resonance Imaging. JAMA Intern Med 173(9): 823825.

8. Willems P (2013) Decision Making in Surgical Treatment of Chronic Low Back Pain: The performance of prognostic tests to select patients for lumbar spinal fusion. Acta Orthop 84(49): 1-37.
9. Papadakis M, Aggeliki L, Papadopoulos EC, Girardi FP (2013) Common surgical complications in degenerative spinal surgery. World Journal of Orthopedics 4(2): 62-66.

10. Nasser R, Yadla S, Maltenfort MG, Harrop JS, Anderson DG et al. (2010) Complications in spine surgery. J Neurosurg Spine 13(2): 144-157.

11. Koes BW, Schoelten RJ, Mens JMA, Bouter LM (1995) Efficacy of epidural steroid injections for low back pain and sciatica: A systematic review of randomized clinical trials. Pain 63(3): 279-288.

12. Wakitani S, Imoto K, Yamamoto T, Saito M, Murata N, et al. (2002) Human autologous culture expanded bone marrow mesenchymal cell transplantation for repair of cartilage defects in osteoarthritic knees. Osteoarthritis and Cartilage 10(3): 199-206.

13. Baksh D, Yao R, Tuan RS (2007) Comparison of proliferative and multilineage differentiation potential of human mesenchymal stem cells derived from umbilical cord and bone marrow. Stem Cells 25(6): 13841392.

14. Chamberlain CS, Saether EE, Aktas E, Vanderby R (2017) Mesenchymal Stem Cell Therapy on Tendon/Ligament Healing. Journal of cytokine biology 2(1):112.

15. Saether EE, Chamberlain CS, Leiferman EM, Kondratko Mittnacht JR, Li WJ, et al. (2014) Enhanced Medial Collateral Ligament Healing using Mesenchymal Stem Cells: Dosage Effects on Cellular Response and Cytokine Profile. Stem cell reviews 10(1): 86-96.

16. Fu Y, Karbaat L, Wu L, Leijten J, Both SK, et al. (2017) Trophic effects of mesenchymal stem cells in tissue regeneration. Tissue Eng Part B Rev 23(6): 515-528.

17. Smith LR (2014) Influencing the secretion of myogenic factors from mesenchymal stem cells. Stem Cell Res Ther 5: 96.

18. De Lisio M, Jensen T, Sukiennik RA, Huntsman HD, Boppart M (2014) Substrate and strain alter the muscle-derived mesenchymal stem cell secretome to promote myogenesis. Stem Cell Res Ther 5: 74.

19. Hofer HR, Tuan RS (2016) Secreted trophic factors of mesenchymal stem cells support neurovascular and musculoskeletal therapies. Stem Cell Research \& Therapy 7(1): 131.

20. Elabd C, Centeno CJ, Schultz JR, Lutz G, Ichim T, et al. (2016) Intradiscal injection of autologous, hypoxic cultured bone marrow-derived mesenchymal stem cells in five patients with chronic lower back pain: a long-term safety and feasibility study. Journal of Translational Medicine 14(1): 253.

21. Pettine K, Suzuki R, Sand T, Matthew Murphy (2016) Treatment of discogenic back pain with autologous bone marrow concentrate injection with minimum two-year follow-up. International Orthopaedics (SICOT) 40: 135.

22. Centeno C, Markle J, Dodson E (2017) Treatment of lumbar degenerative disc disease-associated radicular pain with culture-expanded autologous mesenchymal stem cells: a pilot study on safety and efficacy. Journal of Translational Medicine 15: 197.

23. Chen X, Jones IA, Park C, Vangsness CT (2017) The Efficacy of PlateletRich Plasma on Tendon and Ligament Healing: A Systematic Review and Meta-analysis With Bias Assessment. Am J Sports Med 46(8).

24. Dagenais S, Yelland MJ, Del Mar C, Schoene ML (2007) Prolotherapy injections for chronic low-back pain. Cochrane Database of Systematic Reviews.

25. Zhang J, Middleton KK, Fu FH, Im HJ, Wang JHC (2013) HGF Mediates the Anti-inflammatory Effects of PRP on Injured Tendons. PLos One 8(6): e67303.

26. Enoch S, Price PE (2004) Cellular molecular and biochemical differences in the pathophysiology of healing between acute wounds, chronic wounds, and wounds in the aged. World Wide Wounds 
27. Barrientos S, Stojadinovic O, Golinko MS, Brem H, Tomic Canic M (2008) Growth factors and cytokines in wound healing. Wound Repair Regen 16(5): 585-601.

28. Müller M, Raabe O, Addicks K, Wenisch S, Arnhold S (2011) Effects of non-steroidal anti-inflammatory drugs on proliferation, differentiation and migration in equine mesenchymal stem cells. Cell Biol Int 35(3): 235-248.

29. Breu A, Eckl S, Zink W, Kujat R, Angele P (2013) Cytotoxicity of local anesthetics on human mesenchymal stem cells in vitro. Arthroscopy 29(10): 1676-1684.

30. Binkley JM, Stratford PW, Lott SA, Riddle DL (1999) The Lower Extremity Functional Scale (LEFS): scale development, measurement properties, and clinical application North American Orthopaedic Rehabilitation Research Network. Phys Ther 79(4): 371-383.

31. Liang HW, Hou WH, Chang KS (2013) Application of the modified Lower Extremity Functional Scale in low back pain. Spine (Phila Pa 1976) 38 2043- 2048.

32. Childs JD, Piva SR, Fritz JM (2005) Responsiveness of the numeric pain rating scale in patients with low back pain. Spine (Phila Pa 1976) 30(11): 1331-1334.

33. Khoshbin A, Leroux T, Wasserstein D, Marks P, Theodoropoulos J, et
ISSN: 2574-1241

DOI: 10.26717/BJSTR.2018.07.001461

Marc Darrow. Biomed J Sci \& Tech Res

This work is licensed under Creative Commons Attribution 4.0 License

Submission Link: https://biomedres.us/submit-manuscript.php al. (2013) The efficacy of platelet-rich plasma in the treatment of symptomatic knee osteoarthritis: a systematic review with quantitative synthesis. Arthroscopy 29(12): 2037-2048.

34. Sanchez M, Guadilla J, Fiz N, Andia I (2012) Ultrasound-guided plateletrich plasma injections for the treatment of osteoarthritis of the hip. Rheumatology (Oxford) 51(1): 144-150.

35. Centeno CJ, Al-Sayegh H, Freeman MD, Smith J, Murrell WD, et al. (2016) A multi-center analysis of adverse events among two thousand, three hundred and seventy-two adult patients undergoing adult autologous stem cell therapy for orthopaedic conditions. Int Orthop 40(8): 17551765.

36. Mashaw R (2007) OHRP issues new guidance on unanticipated problems, adverse events. Adverse Event Reporting News 4(2): 1.

37. Kirschner S, Lützner J, Günther K-P, Eberlein Gonska M, Krummenauer F (2010) Adverse events in total knee arthroplasty: Results of a physician independent survey in 260 patients. Patient Saf Surg 4(1): 12.

38. Epstein NE (2013) The risks of epidural and transforaminal steroid injections in the Spine: Commentary and a comprehensive review of the literature. Surgical Neurology International 4(2): S74-S93.

39. Borenstein DG (1996) Chronic Low Back Pain. Rheum Dis Clin 22(3): $439-456$.

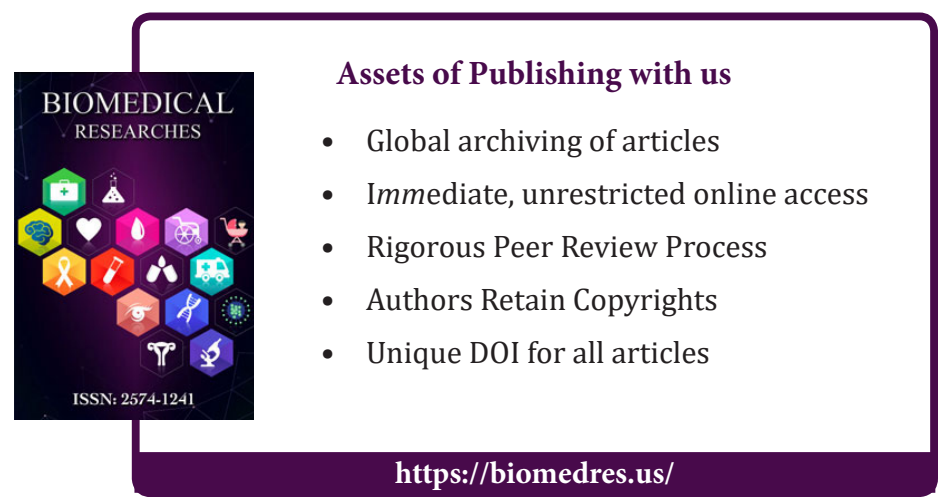

